Théologiques

Théologiques

\title{
Anthropologie du silence
}

\section{David Le Breton}

Volume 7, numéro 2, automne 1999

\section{Silence !}

URI : https://id.erudit.org/iderudit/005014ar

DOI : https://doi.org/10.7202/005014ar

Aller au sommaire du numéro

\section{Éditeur(s)}

Faculté de théologie de l'Université de Montréal

\section{ISSN}

1188-7109 (imprimé)

1492-1413 (numérique)

Découvrir la revue

\section{Citer cet article}

Le Breton, D. (1999). Anthropologie du silence. Théologiques, 7(2), 11-28.

https://doi.org/10.7202/005014ar

\section{Résumé de l'article}

Le silence essentiel à l'écoute nouvelle. Hors du bruit, du grondement d'unecivilisation moderne, du tintamarre quotidien, il est un chemin vers l'écouted'une humanité tapie au fond de l'être. Silence solitaire, silence partagé,silence lourd de sens, à contresens de ce qui voudrait nous éloigner de nousmêmes.Des mots à lire en silence pour se redonner des espaces où mieux sedire se conjugue avec mieux se taire. d'utilisation que vous pouvez consulter en ligne.

https://apropos.erudit.org/fr/usagers/politique-dutilisation/ 


\title{
Anthropologie du silence
}

\author{
David LE BRETON \\ Faculté des Sciences sociales \\ Université de Strasbourg II \\ $M$ ais quand deux hommes s'entretiennent, il y a tou- \\ jours un tiers présent : le silence; il écoute. Ce qui \\ donne de l'ampleur à la conversation, c'est que les \\ paroles ne se meuvent pas dans l'espace étroit des \\ interlocuteurs, mais qu'elles viennent \\ de loin, de là où le silence écoute. \\ (M ax Picard, Le monde du silence)
}

\section{Rumeur du silence}

Le silence ne se confond pas avec l'absence de sonorité, à un monde sans frémissement, étale, ou rien jamais ne se ferait entendre. Le degré zéro du son peut être expérimentalement produit dans un programme de déprivation sensorielle. $M$ ais dans une chambre insonorisée les battements du coeur, la circulation du sang, les mouvements du transit intestinal prennent une ampleur inattendue. Le corps parle de toute sa chair, lui si calme d'ordinaire, devient un univers bruyant, effervescent, empli d'une turbulence inquiétante. Le silence finalement, au sens littéral, n'existeni dans I'hommeni dans la nature. Tout milieu résonne de manifestations sonores particulières, même si elles sont espacées, ténues, étouffées, lointaines, à la limite de l'audible. Les étendues désertiques ou les hautes montagnes ne sont jamais tout à fait muettes, encore moins les forêts ou les campagnes. Les cours de monastères elles-mêmes sont bruissantes de la cloche qui sonne, des chants liturgiques ou des prières émanant de l'église. Les mouvements de l'homme dans l'espace laissent la trace sonore de ses pas, de ses gestes, de son souffle ; son immobilité même n'annule pas sa respiration et les bruits de son corps. Toujours l'existence palpite et fait entendre sa rumeur. $M$ ême dans le calme du soir celui qui écoute 
attentivement et se laisse bercer par les lieux entend l'herbe pousser ou les mouvements fugitifs et incessants des fourmis.

La campagne est davantage associée au silence dans le discours des citadins, mais par opposition au bruit généré par la ville. Car là aussi la rumeur tranquille du monde ne s'interrompt jamais, changeant seulement de nature d'un lieu et d'un temps à l'autre, et selon les saisons. Le vent agite les feuilles et les branches, les troncs craquent, des animaux se meuvent discrètement sous les fourrés ; le bruissement des rivières ou des sources n'a d'autre repos que leur assèchement. Parfois l'apaisement de la nuit porte au loin des éclats de voix, une querelle de chats, les aboiements d'un chien ou le passage d'une voiture dans la forêt proche. L'obscurité, même dans les lieux retirés, n'est pas un monde de pierre où les sens seraient mis en pénitence. Au coeur de la fermeles meubles travaillent et leurs craquements effraient parfois dans l'immobilité apparente des lieux. Les cendres achèvent de se consumer, provoquant parfois un effondrement dans l'âtre. Au sein des maisons, le ronronnement des équipements électriques, les bruits du chauffage, des conduites d'eau, de la rue voisine, des appareils de radio ou de télévision, les voix, les déplacements des habitants du lieu rendent l'espace vivant, chaleureux. Si l'impression du silence n'en demeure pas moins, elle est plutôt l'effet d'une interprétation affective des lieux qu'une mesure rigoureuse des faits.

Des sons se mêlent au silence sans en déranger l'ordonnance. Parfois même ils le révèlent et éveillent alors I'attention à la qualité de présence d'abord inaperçue d'un lieu. Le silence est d'abord une modalité du sens, un sentiment qui saisit l'individu. M ême si le bruissement du monde ne s'arrête jamais, connaissant seulement des variations différentes au gré des heures, certains lieux n'en donnent pas moins le sentiment d'une approche du silence : une source se frayant un chemin parmi les pierres, l'ululement d'une chouette au coeur de la nuit, la cloche d'une église égrenant les heures à la tombée du soir, le crissement de la neige sous les pas, le craquement d'une pomme de pin sous le soleil, donnent une épaisseur au silence. Ces manifestations légères accentuent le sentiment de paix qui émane du lieu. C e sont des créations du silence, non par défaut mais parce que le spectacle du monde n'y est recouvert d'aucun parasite, d'aucun bruit. "II semble, dit Bachelard, que pour bien entendre le silence notre âme ait besoin de quelque chose 
qui se taise $\gg .{ }^{1}$ Le silence sonne comme la signature d'un lieu, substance presque tangible dont la présence hante l'espace et s'impose constamment à l'attention. Albert Camus, cheminant parmi les ruines de $D$ jémila, observe un « grand silence lourd et sans fêlure, quelque chose comme l'équilibre d'une balance. Des cris d'oiseaux, le son feutré de la flûte à trois trous, un piétinement de chèvres, des rumeurs venues du ciel, autant de bruits qui faisaient le silence et la désolation de ces lieux $\gg .^{2}$ Ce n'est pas la disparition des sons qui fait le silence, mais la qualité de l'écoute, la pulsation infime d'existence qui anime l'espace. Le saut d'une carpe sur les eaux étales du lac est le plus bel hommage au silence. ${ }^{3}$

Dans le contexte éminemment sonore de la modernité le silence se donne comme une absence de bruit, un horizon encore épargné par la technique, zone provisoirement en friche non encore absorbée ou délibérément conçue comme une réserve de silence. Le monde résonne sans relâche des instruments techniques dont l'usage accompagne la vie personnelle ou collective. La modernité est l'avènement du bruit. Le seul silence que nos sociétés connaissent est celui, provisoire, de la panne, de la défaillance de la machine, de l'arrêt de transmission. Il est une cessation de la technicité plutôt que l'émergence d'une intériorité. II suffit parfois que s'éteigne un bruit continu, que le moteur de la voiture s'interrompe un instant ou que la pause disperse les ouvriers du chantier pour que lesilence se donneà portée de la main, d'une présence sensible à la fois matérielle et volatile. $M$ archant dans le $D$ olpo, une région du N épal frontalière du Tibet, P. M athiessen et son compagnon $G$. Schaller ont soudain la révélation du silence où ils baignent depuis leur arrivée dans ces lieux. " Vous rendez-vous compte que depuis septembre nous n'avons pas entendu un seul bruit de moteur, même éloigné, me dit GS. Et c'est vrai. A ucun avion ne franchit ces vieilles montagnes. $\mathrm{N}$ ous nous sommes aventurés dans un autre siècle ${ }^{4}{ }^{4}$ Le silence renvoie alors à une expérience antérieure à la technique, à un univers sans moteur, sans voiture, sans avion, le vestige archéologique menacé d'un autre temps. Et la marche lente du retour est difficile et amère car elle

1. G. BACHELARD, L'eau et les rêves. Essai sur l'imagination de la matière, Paris, Corti, 1942, p. 258.

2. A. CAm US, N oces, Paris, Livre de poche, 1959, p. 25.

3. Voir D. LE BRETON, D u silence, Paris, M étailié, 1997.

4. P. M AthiesSen, Le léopard des neiges, Paris, Gallimard, 1983, p. 110. 
est une progression vers le bruit après des mois de paix intérieure. «En longeant les collines de la Bheri cet après-midi, je me suis rappelé l'importance de ne pas trop parler, de ne pas bouger trop brusquement après une semaine de retraite zen et de silence... II est capital de n'émerger que progressivement de cette chrysalide, de faire sécher ses ailes encore humides dans le calme, au soleil, comme un papillon, pour éviter un déchirement psychique trop brutal » (p. 321). L'espacen'est pas uniquement fait de ce que l'homme voit, mais aussi de ce qu'il entend. Un univers où règne le silence ouvre une dimension particulière au sein du monde. A près ces mois de silence, il importe de ne pas se hâter, de marcher lentement vers la vallée, de se laisser porter par les heures sans les précipiter. Comme un plongeur des hauts fonds, le voyageur encore baigné de silence marque des paliers pour ne pas être heurté de plein fouet par le vacarme à venir de la vie sociale.

La recherche du silence traduit une volonté d'apaisement, de recueillement, d'immersion dans un lieu propice. II manifeste un gisement moral dont le bruit seul est l'ennemi mortel, il signe une interprétation par l'individu de ce qu'il entend, et une voie de repli sur soi pour retrouver le contact avec le monde. $\mathrm{M}$ ais il requiert parfois l'effort de le trouver, de le débusquer dans une démarche volontaire. "L'autre soir, écrit Thoreau, j'étais décidé à mettre un terme à ce vacarme futile, à marcher dans différentes directions pour voir si on ne pouvait pas trouver silence aux alentours... Je quittai le village pour remonter en bateau la rivière jusqu'au lac de Fair $\mathrm{H}$ aven... La rosée en train de se déposer semblait filtrer, tamiser l'air et je me sentis apaisé par le calme infini. Voilà qu'en quelque sorte je tenais le monde par la peau du cou, le maintenant sous le courant de ses propres éléments jusqu'à ce qu'il se fût noyé. Je le laissai ensuite partir avec le flot comme un chien mort. D'immenses espaces de silence s'étiraient de tous côtés, et mon être s'épanouissait en proportion pour les remplir. Ce n'est qu'ensuite que je pus pour la première fois apprécier le bruit et le trouver musical $\gg .5$

\section{Recueillement}

Les lieux de culte ou les jardins publics, les cimetières, forment dans les villes des enclaves de silence cernées par le bruit où il est loisible de

5. Cité dans H enry D. Thoreau, Paris, L'H erne, 1994, p. 39-40. 
chercher un repos, une brève retraite hors du tumulte ambiant. O n vient y reprendre souffle, se recueillir, goûter le calme que berce le genius loci. Le silence installe dans le monde une dimension propre, une épaisseur qui enveloppe les choses. Le temps y passe sans hâte, à pas d'homme, appelant le repos, la méditation, la flânerie. Ces lieux sertis de silence se détachent du paysage en se donnant d'emblée comme propices au rassemblement de soi. O n y fait provision d'intériorité avant le retour aux agitations de la ville ou de sa propre existence. ${ }^{6}$

Le silence pénétrant d'un bâtiment ou d'un paysage est un chemin menant à soi. M oment de suspension du temps. Provision de sens et de force intérieure avant le retour au vacarme du monde et aux soucis du quotidien. Le pointillé du silence goûté à différents moments de l'existence par le recours à la campagne ou au monastère, au désert ou à la forêt, ou simplement au jardin, au parc apparaît comme un ressourcement, un temps de repos avant de retrouver le bruit, entendu au sens propre et au sens figuré, d'une immersion dans la civilisation urbaine. Le silence procure alors un sentiment aigu d'exister. II marque un moment de dépouillement qui autorise à faire le point, à prendre ses marques, à retrouver une unité intérieure, à franchir le pas d'une décision difficile. Le silence élague l'homme et le rend à nouveau disponible, déblaie le chantier au sein duquel il se débat. Le promeneur attentif entre lentement par son écoute dans ses différents cercles, à chaque instant il pénètre d'autres univers sonores qui peuplent l'épaisseur du silence. II se découvre soudain un sens nouveau, non l'approfondissement de l'ouïe, mais un sens inédit attaché à la perception du silence.

«II y a le matin qui se lève sans bruit, il y a les arbres qui se tendent sans bruit vers le ciel et il y a le soir qui tombe comme à la dérobée». ${ }^{7}$ Certains lieux rendent impensable l'effraction d'un son étranger ou d'une parole bavarde, on y marche dans la crainte de rompre un équilibre fragile qui ne se prête pas à l'intervention sensible de I'homme sinon à la contemplation. D ans la forêt, le désert, la montagne ou la mer, le silence pénètre parfois si parfaitement le monde que les autres sens deviennent désuets ou inutiles. La parole est sans voix pour dire la puissance de l'instant ou la solennité des lieux. Kazant-

6. Sur les aspects religieux ou la dimension d'intériorité du silence, voir D. LE BRETON, D u silence, p. 176 ss.

7. M. PICARD, Le monde du silence, Paris, PUF 1953, p. 169. 
zaki marche avec un ami au coeur d'une forêt du M ont A thos sur le chemin pavé de Karyès. « II semblait que nous étions entrés dans une immense église : la mer, des forêts de châtaigniers, des montagnes et par-dessus, en guise de coupole, le ciel ouvert. Je me suis tourné vers mon ami - Pourquoi ne parlons nous pas?, dis-je, voulant rompre le silence qui commençait à me peser ». - « N ous parlons, répondit mon ami, en me touchant légèrement l'épaule, nous parlons, mais la langue des anges, le silence ». Et brusquement, comme s'il s'était mis en colère : «Q ue veux-tu que nous disions? Quec'est beau, que notre coeur a des ailes et veut s'en aller, que nous avons pris le chemin qui mène au Paradis? D es mots, des mots! Tais-toi $\gg .{ }^{8}$ Le silence partagé est une figure de la complicité, il prolongel'immersion dans la sérénité de l'espace. Le langage réintroduit la séparation qu'il cherche à conjurer sans jamais y parvenir tout à fait. Le recueillement butte contre une parole qui le dissipe par l'attention qu'elle provoque. Le dialogue est alors arrachement au paysage, infidélitéau genius loci, satisfaction donnée aux normes sociales et manière conventionnelle de se rassurer ou de sortir de son isolement émerveillé. L'émotion se perd dans le mouvement où elle s'énonce. « La suite naturelle de la contemplation serait le silence, écrit Brice Parain. Sous le coup de cette puissance gigantesque, qui m'attire et m'effraie à la fois, il me faudrait quelque temps pour m'en délivrer, pour ne plus me sentir écrasé, vaincu, fasciné ... Les mots que je pourrais prononcer me paraîtraient une mauvaise revanche, même s'ils étaient pour admirer ou révérer». ${ }^{9}$ Le sentiment d'alliance avec le cosmos, de dissolution de toutes limites relève d'un sacré intime à la merci du moindre bavardage. II faut savoir se taire pour ne pas briser le vase infiniment fragile du temps.

La conjugaison du silence et de la nuit est également propice à l'immersion de soi dans la sérénité des lieux. L'obscurité, à peine entamée par une lumière vacillante, éveille chez J ames A gee un vocabulaire religieux, étranger pourtant à sa sensibilité mais qui s'impose soudain, dessinant dans l'espace une scène qu'on dirait peinte par de La Tour. La flamme de la lampe derrière le verre « a la délicatesse sèche, silencieuse, famélique - des extrémités tardives de la nuit, une

8. N. KazAntZakı, Lettre au G réco, Paris, Plon, 1961, p. 189-190.

9. B. Parain, Petite métaphysique de la parole, Paris, Gallimard, 1969, p. 20. 
délicatesse d'un silence et d'une paix si ultimes et si saints que tout sur terre et jusqu'aux confins les plus éloignés de la remémoration y semble suspendu à la perfection comme dans le miroir des eaux : et je ressens que si dans une quiétude entière je peux parvenir à ne pas troubler ce silence, comme en m'astreignant à ne pas toucher seulement à cette plaine des eaux, je peux tout vous dire au royaume de Dieu, quoique ce soit que l'envie me vienne de vous dire, et quoique ce soit, vous ne pourrez pas vous empêcher de le comprendre». ${ }^{10} \mathrm{Le}$ recueillement est l'une des modalités que le silence prodigue à ceux qui s'établissent un moment en lui. R etour sur soi, capacité de se laisser envahir par le paysage ou la solennité des lieux.

Le silence est alors un baume qui guérit de la séparation avec le monde, avec les autres, avec soi : il restaure symboliquement l'unité perdue que la résurgence du bruit anéantit à moins d'avoir la force de faire le silence en soi en dépit des rumeurs avoisinantes. II laisse à I'homme la possibilité de se laisser envahir par la solennité des lieux, porté par le frémissement de l'atmosphère. "II semblait que la matinée se fût fixée, le soleil arrêté pour un instant incalculable, écrit $C$ amus. D ans cettelumière et ce silence, des années de fureur et de bruit fondaient lentement. J'écoutais en moi un bruit presque oublié, comme si mon coeur, arrêté depuis longtemps, se remettait doucement à battre ». Le silence met le monde en suspens, il maintient l'initiative de l'homme en le laissant respirer dans le calme d'un souffle que rien ne presse. II est retrouvailles avec le sens, il restaure le sentiment de la présence au monde.

\section{La peur du silence}

L'écrivain J. A bbey s'installe dans un désert de l'O uest américain. "Seul dans le silence, je comprends un instant I'effroi que beaucoup éprouvent en présence du désert primordial, la peur inconsciente qui les pousse à domestiquer, altérer ou détruire ce qu'ils ne peuvent pas comprendre, à réduire le sauvage et le préhumain à des dimensions humaines. Tout plutôt que d'affronter directement le préhumain, I'autre monde qui n'effraie pas par le danger ni l'hostilité, mais par quelque chose de pire : son implacable indifférence $»{ }^{11}$ L'homme est guetté par

10. J. Agee, W. Evan S, L ouons maintenant les grands hommes, Paris, Plon, 1972, p. 67.

11. J. A BBEY, D ésert solitaire, Paris, Payot, 1997, p. 273-274. 
le silence, il se sent captif, saisi par une forme immatérielle qui pèse sur ses paroles ou ses gestes et réfrène son action. Souvent le silence appelle l'inquiétude car il ouvre une métaphysique du lieu ou de la présence de I'autre. II y a une sorte d'écoute du silence, un oeil invisible qui renvoit à une intériorité exigeante. Si certains individus s'établissent en lui comme en un refuge et y trouvent un lieu propice à un retour sur soi, d'autres s'en effraient et n'ont de cesse de s'en défendre. ${ }^{12}$ " D'où provient l'inquiétante étrangeté qui émane du silence, de la solitude, de I'obscurité?, s'interroge Freud... N ous ne pouvons rien dire, si ce n'est que ce sont là vraiment les éléments auxquels se rattache l'angoisse infantile qui jamais ne disparaît tout entière chez la plupart des hommes ». ${ }^{13} \mathrm{O}$ tto, de manière proche, observe que l'art occidental ne dispose que de deux moyens, I'un et l'autre négatif, pour faire référence au numineux : le silence et l'obscurité. ${ }^{14}$

La question du silence soulève celle de l'ambivalence du sacré, elle livrel'hommeà l'ambiguïté. Le sacréarracheà l'ordinaire de l'existence en procurant une plage de temps ou d'espace saturée d'être. II cristallise une valeur, une différence sensible qui hiérarchise subtilement des moments ou des objets particuliers (une maison, un jardin, la nuit, le silence, la fête, etc.). Le mysterium tremendum du numineux est ce qui fait frissonner l'homme et lui fait ressentir la fragilité de sa condition. « Le sentiment qu'il provoque peut se répandre dans l'âme comme une onde paisible, note $\mathrm{O}$ tto ; c'est alors la vague quiétude d'un profond recueillement », manifestant « le silencieux et humble tremblement de la créature qui demeure interdite en présence de ce qui est dans un mystère ineffable, au-dessus de toute créature». ${ }^{15} \mathrm{M}$ ais l'autre versant est celui de l'effroi devant cette condition, l'impression de se perdre face à une présence écrasante et inintelligible. La relation au silence appelle ainsi, sel on les circonstances et les individus, la paix ou l'angoisse.

12. Sur la peur du silence dans les conversations, le fait qu' « un ange passe » et sur les différences de régimes culturels de parole et de silence dans l'échange avec autrui, voir D. LE BRETON, D u silence.

13. S. FREUD, L'inquiétante étrangeté, Essai de psychanalyse appliquée, Paris, Gallimard, 1971, p. 202 et 210.

14. R. O TTO, Le sacré, Paris, Payot, 1969, p. 107.

15. Ibid., p. 28. 
«Et lorsquel'A gneau ouvrit le septième sceau, il se fit un silence dans le ciel, environ une demi-heure », dit l'A pocalypse de Jean. Si le silence résonne soudain comme une rupture du bruissement coutumier du monde, il induit l'angoisse. L'ennemi est là, dans les ténèbres, qui avance et les animaux se taisent, le vent lui-même suspend son souffle, l'avancée du crime et de la mort semble aller de pair avec un monde en attente, aux aguets de l'irréparable, et ce bruyant silence est une alerte lancée au sens de l'homme avisé qui ne perçoit rien de naturel dans l'effacement brutal des sons. II signe alors la marque tangible d'un danger qui se ramasse sur soi pour fondre bientôt sur sa proie. Rilke traduit la même expérience à Paris. Alors qu'il vient de stigmatiser le bruit, il enchaîne soudain sur «quelque chose qui est plus terrible: le silence. Je crois qu'au cours de grands incendies il doit arriver, ainsi, parfois, un instant de tension extrême : les jets d'eau retombent, les pompiers ne montent plus à l'échelle, personne ne bouge. Sans bruit, une corniche noire s'avance, làhaut, et un grand mur derrière lequel le feu jaillit s'inclinesans bruit. Tout le mondeest immobile et attend, les épaules levées, le visage contracté sur les yeux, le terrible coup. Tel est ici le silence ». ${ }^{16}$ Image de mort, force colossale qui s'apprête à broyer l'homme

Si les uns, comme James Agee, cité ci-dessus, connaissent au milieu du silence et de la nuit le sentiment de baigner dans une paix que rien ne trouble, d'autres s'inquiètent d'un tel calme dont ils craignent qu'il ne couve le pire. "Je n'aime pas ce silence, il ne présage rien de bon » disaient les éclaireurs ou les soldats des films de notre enfance. La qualité particulière de sonorité qui émane de la nuit, en supprimant le murmure rassurant des activités diurnes est propice à l'émergence du pire ou du meilleur, l'angoisse ou le recueillement se mêlent ou se succèdent. La nuit confère au silence une puissance accrue en effaçant les contours du monde, en renvoyant provisoirement (mais qui peut savoir quelle en sera la durée quand l'angoisse est là) le connu à l'informe, au chaos. Le monde est en suspens, noyé dans une obscurité recelant toutes les menaces aux yeux de qui est plongé dans l'effroi. II semble que le silence et la nuit mêlés préparent une offensive contre le jour et la parole, brisant les ultimes ressources d'humanité, privant I'homme d'orientation, le livrant à lui-même, à l'épreuve redoutable de sa liberté. Ils lui imposent une conscience de

16. R. M . RILKE, L es cahiers de M alte L aurids B rigge, Paris, Seuil, p. 12-13. 
son inachèvement. $D$ ans la terminologie biblique, la racine damô ${ }^{17}$ associe étroitement le silence, la nuit et la mort. «Et c'est par le silence que la mort évoque la nuit, écrit-il, comme inversement la nuit ressemble à la mort par le silence. Si la nuit et la mort sont intuitivement éprouvées comme étant de la même famille, si la nature de l'une fait immédiatement penser à l'autre, si les poètes dans leurs métaphores, les mystiques dans leurs prières, les misérables dans leurs cris, peuvent indifféremment s'adresser à l'une ou à l'autre avec la certitude de faire vibrer une seule et même corde, c'est parce que la nuit et la mort sont toutes deux silencieuses $\gg\left(\right.$ p. 42). ${ }^{18}$

Le silence annule toute diversion et met l'homme face à soi, le confronte aux douleurs enfouies, aux échecs, aux repentirs, à l'interrogation sur ce que recouvre sa venue. Il ôte toute prise sur l'événement et suscite la peur, l'effondrement des repères qui amènent par exemple des citadins endurcis à ne pouvoir s'endormir dans une campagne ou une maison silencieuse. La nuit accroît le malaise en privant de la sécurité visuelle que procure le jour, elle ouvre les vannes à l'ambiguïté du monde. A ux aguets, ils perçoivent sur cette toile de fond le moindre frémissement du dehors ou le craquement d'une armoire comme autant de menaces. II leur faut s'accoutumer au calme des environs, apprivoiser les sons qui les entourent et cesser de voir l'absence de bruit comme un mode d'approche sournois de l'ennemi. Le silence relâche l'emprise du sens, il désoriente les repères et restitue l'initiative à l'individu. II exige de posséder les ressources symboliques pour en jouir sans céder à la peur sinon, à l'inverse il ouvre les vannes du fantasme. "Lorsque l'homme se trouve seul, éloigné du tumulte des villes, écrit $M$ arie-M adeleine Davy, il perçoit les voix des bêtes sauvages qui font en lui litière. II sursaute en éprouvant une certaine panique difficile à surmonter. En effet, il ignorait nourrir en lui-même les animaux dont il perçoit les clameurs ». ${ }^{19}$

17. A. NeHER, L'exil de la parole. Du silence biblique au silence d'Auschwitz, Paris, Seuil, 1970, p. 39.

18. A. N eher observe encore que s'il lui fallait ramasser le contenu de cette forme biblique particulière du silence, il proposerait le terme d' « inertie», « voulant désigner par là non point la passivité intrinsèque de cet univers de silence, mais sa négativité par rapport à l'homme. Le silence-inertie désigne un cosmos qui a, sans doute, ses lois et ses mouvements propres, mais le secret de ces lois et de ces mouvements est impénétrable à l'homme » (p. 43).

19. M -M . DAvY, « Le silence intérieur » Corps écrit, n¹2, 1984, p. 170. 
Le silence favorise un retour du refoulé quand le rempart du sens que fournit le bruit se dérobe en partie, il semble ronger la parole à sa source et la rendre impuissante. D'où le cri, évoqué par Freud, d'un enfant de trois ans couché dans une chambre sans lumière: "Tante, dis-moi quelque chose, j'ai peur, parce qu'il fait si noir ». La tante lui répond : «A quoi cela te servira-t-il puisque tu ne peux pas me voir? ». «Ca ne fait rien, répondit l'enfant. Du moment que quelqu'un parle, il fait clair». ${ }^{20} \mathrm{~L}$ a parole familière est une objection au silence angoissant del'environnement, à la suspension inquiétante des repères laissant suspecter un sol qui se dérobe sous les pas. Le silence est en effet également associé au vide de sens et donc à la menace d'être englouti dans le néant. La parole est alors ce filet de signification, le pointillé d'une présence peuplant le monde de son humanité rassurante. Dans la rumeur indifférente du réel une voix introduit un centre, elle organise le sens autour d'elle.

Le silence ouvre à la profondeur du monde, il découpe une autre dimension au sein du réel, il force à la métaphysique en soustrayant les choses au murmure qui les enveloppe d'ordinaire et libère ainsi leur puissance contenue. II prive des repères sécurisants qui apaisent la relation aux objets ou aux autres en confrontant l'homme à la concrétude de faits dont il découvre combien ils lui échappent finalement, combien le sens qui rend l'univers familier n'est qu'une convention nécessaire, mais si fragile qu'un rien ne la désagrège, surface heureuse d'évidences qui fait oublier le vide qu'elles renferment ou le mystère plutôt qu'elles cherchent à atténuer. Le rapport au silence est une épreuve qui révèle des attitudes sociales et culturelles, mais aussi personnelles de l'individu. Certains s'effraient d'un monde ainsi mis à nu où disparaissent les traces sonores qui tapissaient leur tranquillité d'esprit en rendant leur existence habitable et compréhensible. Ils voient dans le bruit une étoffe de sens les protégeant de la brutalité du monde, bouclier contre le vide qu'appelle à leurs yeux le silence. Et l'événement existe en effet par l'intrusion de son bruit, il entaille le silence qui donne au contraire le sentiment d'une étendue plane, sans défaut, sans histoire, à la fois emplie de sécurité et d'angoisse à cause de son absence de limite et de sa polysémie. Le bruit s'identifie tou-

20. S. Freud, Trois essais sur la théorie de la sexualité, Paris, Gallimard, p. 186. 
jours assez clairement à une source, le silence inonde l'espace et laisse la signification en suspens à cause de ce pouvoir ambigu de traduire mille choses à la fois.

\section{L'exorcisme du bruit}

Ceux qui craignent le silence demeurent à l'affût d'un son qui humaniserait les lieux, ils s'inquiètent de parler comme si leur parole allait mettre en branle des forces obscures prêtes à fondre sur eux. D'autres, pour échapper à l'angoisse, se lancent dans des actions bruyantes, crient ou sifflent, s'accompagnent d'une radio ou d'un magnétophone. Leur manifestation ostentatoire efface une situation insupportable. En restaurant l'empire du bruit, ils cherchent à rétablir les droits d'une humanité en suspens, ils retrouvent leurs assises identitaires un moment ébranlées par l'absence de tout repère sonore identifiable. Le bruit exerce en effet une fonction sécurisante en dispensant des signes tangibles d'existence, en témoignant de la turbulence sans fin d'un monde toujours présent. II donne prise, surtout si on en est soimême le maître, là où le silence à l'inverse est insaisissable et dépasse à l'infini l'individu. La radio ou la télévision peuplent la maison, et restent parfois en marche comme simple bruit de fond conjurant la solitude, le silence, le deuil, le vide d'une existence sur le fil du rasoir. Le bruit humanise les lieux, les rend retentissants de la présence des autres.

O pposé au silence, le bruit a souvent une fonction propitiatoire dans les usages traditionnels, et même encore aujourd'hui lors de certaines transitions sociales. Les conduites de vacarme ont longtemps accompagné les noces de maintes régions européennes. La pratique subsiste avec le cortège de voitures qui traverse ville ou campagne à grand renfort de klaxon. $F$. Z onabend ${ }^{21}$ décrit les noces à $M$ inot, dans le Châtillonnais, en insistant sur le tapage rituel qui jalonne la cérémonie. Bruits et cris au fil du parcours, appels des enfants, cloches, coups de fusil, klaxon, etc. Ponctué de rires, d'acclamations, de cris, de chansons, etc. le repas de noces dure des heures. Les habitants de $M$ inot s'étonnent qu'aujourd'hui des noces soient parfois silencieuses : "Les gens ne savent plus s'amuser, il y a des noces maintenant, on n'entend rien ». La suspicion pèse sur ces mariages : conflits entre les parents, conduite inconvenante de la mariée? Ce sont là en principe, selon F.

21. F. Zon Abend, La mémoire longue, Paris, PUF, 1980, p. 180 ss. 
Zonabend, les motifs traditionnels de noces célébrées dans la discrétion, sans fracas d'armes ou de pétards, sans chants, sans manifestations tapageuses. $M$ anière de ne pas faire de bruit au sens figuré, pour ne pas se désigner à l'attention. Le tintamarre rituel de la noce affiche la liesse et affirme publiquement le mariage, mais il participe aussi du changement de statut de la jeune femme, «disjonction pleine de risques » que le vacarme accompagne et symbolise, selon Levi-Strauss, en éloignant les instances négatives et en appelant la fécondité, l'abondance sur le couple. Le silence lors du rituel serait signe de stérilité, d'un danger, ou l'aveu implicite d'une conduite coupable. ${ }^{22}$

C. Levi-Strauss pointe également le tapage mené par certaines sociétés traditionnelles à l'occasion des éclipses, manière de signaler là aussi « une anomalie dans la chaîne syntagmatique » (p. 295). M. Godelier décrit un tintamarre rituel chez les Baruya de N ouvelle-Guinée au moment d'une éclipse : « J e compris à travers ces cris que la lune était « en train de mourir ». A ussitôt après que ces mots eurent étécriés, monta de toutes les directions du village un charivari de bruits d'objets qu'on frappe et de clameurs poussées. A près une longue minute, le silence revint $\gg .{ }^{23}$ Ces conduites acoustiques de conjuration d'une menace par le bruit se retrouvent dans maintes fêtes calendaires des traditions européennes et de manière moderne au moment du changement d'année qui voit dans les villes des concerts de klaxons et l'explosion d'innombrables pétards, de feux d'artifice, etc. ${ }^{24}$

O n peut aussi fuir le silence comme la peste dans une quête passionnée de saturation auditive. Le bruit revêt alors une signification propice pour l'individu ou le groupe, il construit un paravent permettant le retrait hors du monde et la prévention de contacts non désirés. La culture du baladeur pousse à son terme ce souci de s'isoler dans la continuité d'une sonorité inlassable qui rend simultanément encore plus intolérable toute confrontation ultérieure au silence. $R$ ien n'est plus vulnérable que le silence, s'il en demeure quelques nappes ici et

22. C. Levi-Strauss, Le cru et le cuit, Paris, Plon, 1964, p. 293.

23. M . Godelier, dans J. Le G off, J-C. SCH M ITT (éds), Le charivari, Paris, M outon, 1981, p. 347.

24. A l'inverse à Bali où règne un silence d'autant plus formidable que l'existence coutumière est souvent très bruyante, voir M.M EAD, G. BATESON, Balinese Character, N ew York A cademy of Science, 1942, p. 1 ss. 
là, tout individu dispose des moyens techniques de s'en défendre s'il le souhaite. D'où l'usage inattendu du baladeur lors d'activités comme le jogging ou la randonnée; le transistor ou la radio dans la voiture en marche, la portière ouverte, dans des lieux plutôt associés au repos, à la tranquillité sonore: les plages par exemple, les campagnes, les abords des lacs fréquentés par les baigneurs ou les pêcheurs, etc. Si certains se réfugient dans le silence, d'autres lui préfèrent le bruit, ils y trouvent les mêmes ressources pour le rassemblement de soi, la protection contre un environnement perçu comme hostile ou étranger, la conjuration de l'angoisse, de la solitude. Le bruit autorise également une structuration identitaire. II n'est pas un seuil sonore ou un contenu unanime, mais une signification donnée par l'individu, c'est-àdire aussi un jugement de valeur. Le son heureux de l'un : le moteur d'un camion ou la musique d'un haut-parleur poussée à la limite, est l'inconfort ou le désespoir de l'autre.

La muraille sonore érigée par l'autoradio ou le CD, la discothèque, le baladeur, ou la salle de concert avec une sono portée à son comble, isole d'un monde difficile à saisir en donnant une sécurité provisoire, un sentiment de contrôle sur l'environnement. Le bruit au sein du groupe de pairs entrave parfois la communication, la réduit à une pure forme phatique mais elle empêche aussi de trop mesurer solitude ou désarroi. Elle favorise sans effort le sentiment de communion. La recherche de maîtrise par la production du fracas ou du retrait sonore engendre aussi le plaisir, il est un mode efficace de gestion identitaire, un élément de la constitution de soi comme sujet. « Le monde extérieur, écrit $\mathrm{G}$. Steiner, se réduit à un jeu de surfaces acoustiques». ${ }^{25}$ L'individu glisse avec son baladeur d'une ambiance sonore à une autre, bien arrimé à un univers hospitalier où il a prise et dont il contrôle toutes les données. Il efface ainsi les aspérités du monde. Le bruit donne un signe tangible de la présence des autres près de soi. II rassure en rappelant qu'au delà de soi le monde continue à exister.

Pourtant le bruit est parfois lui-même messager del'angoisse quand il rompt inopinément le silence. Le craquement du parquet dans la maison que l'on croyait vide, un bruit de pas dans le jardin pourtant clos, un cri dans la campagne déserte, un frémissement dans les fourrés

25. G. STEIN ER, L e château de Barbe-Bleue. N otes pour une redéfinition de la culture, Paris, Gallimard, 1973, p. 132. 
manifestent une intrusion inquiétante. Effraction de l'événement dont le bruit déchire le silence et éveille une image de mort. Ces manifestations sonores insolites qui dissolvent la paix environnante apparaissent comme des glissements projetant l'homme "à la lisière de l'autre monde », le mettant en position d'en recevoir un message, voire même " d'y être entré sans (s)'y être dissous, ou bien d'embrasser du regard la marche de la vie et de la mort selon une optique d'outre-tombe». ${ }^{26}$ Dans l'épaisseur étale du silence, on conçoit combien la déchirure du bruit est une menace, un rappel de la fragilité et de la finitude qui saisit I'homme et lui impose de se tenir sur ses gardes.

\section{M odernité}

À la profusion du bruit né de la ville, à la ronde incessante des voitures, nos sociétés ajoutent de nouvelles sources sonores avec les musiques d'ambiance dans les magasins, les cafés, les restaurants, les aéroports, etc., comme s'il fallait noyer le silence des lieux où la parole s'échange à l'intérieur d'un bassin permanent de bruits que nul n'écoute, qui indispose parfois, mais dont l'intérêt est de distiller en sous main un message sécurisant. Antidote à la peur diffuse de n'avoir rien à dire, infusion acoustique de sécurité dont la rupture soudaine suscite une gêne redoublée. La musique d'ambiance est devenue une arme efficace contre une certaine phobie sociale du silence. Cet univers sonore insistant isole les conversations particulières ou envel oppe les rêveries, confine chacun dans un espace propre, équivalent phonique des paravents enfermant les rencontres sur elles-mêmes, créant une intimité par le brouillageainsi suscité autour de soi. Le retour du silence, en fin de bande par exemple, rend sensible aux paroles échangées, à leur contenu, elle brise la discrétion antérieure, elle interdit la rêverie, et refrène même les pauses dans les discussions de crainte que ces moments ne soient confondus avec un vide ou une indifférence. II est plus facile de se taire en présence d'une musique d'ambiance que dans le silence d'une salle d'attente où l'effacement ritualisé du corps, notamment, est plus malaisé à accomplir, la gêne plus tangible à moins de s'oublier dans la lecture d'une revue ou d'un livre et de parvenir à faire le silence en soi. ${ }^{27}$

26. M. LEIRIS, Fourbis, Paris, Gallimard, 1955, p. 23.

27. D. LE BRETON, Anthropologie du corps et modernité, Paris, PUF, 1990, p. 125 ss. 
En le coupant de ses sources originelles par l'enregistrement et en recourant aux instruments appropriés le son bénéficie d'un usage sans limite. ${ }^{28}$ Une fois fixé il peut être entendu maintes et maintes fois sans que l'individu sorte de chez soi, sans que l'orchestre se remette à l'ouvrage. Un même chant d'oiseau ou de baleine est disponible pour l'amateur même après avoir été recueilli de longues années auparavant. On peut entendre la voix d'un proche bien après sa mort. Une immense phonothèque est sans relâche à la disposition de l'amateur. Le tumulte du monde est susceptible de peupler sa chambre à toute heure du jour ou de la nuit. Les sons sont reproductibles à l'infini, on peut même imaginer qu'ils survivent longtemps à la disparition des hommes. La modernité a inventé la constance de la sonorité et la capacité de la démultiplier par les haut-parleurs. L'individu qui ne supporte pas le silence a le loisir de recourir dans l'ensemble des faits et gestes de la vie quotidienne à un bruit de fond. Les programmes de la radio ou de la télévision ne s'arrêtent jamais, ni les environnements musicaux banalisés des espaces publics, des halls d'hôtels, des cafés, des boutiques, des restaurants, des galeries commerciales, parfois même des moyens de transport. A rrachée à ses racines de silence, la parole elle-même se dégrade en bruit de fond. Une litanie sans fin accompagne l'homme au fil du jour, lui fournissant sans relâche des repères sécurisants. Et s'il entre chez lui dans le silence relatif de sa demeure, il lui revient d'allumer sa radio ou sa télévision, de regarder une vidéo ou d'écouter cassettes ou CD. Le bruit exerce un effet narcotique au sein de l'appartement ou dans la rue, il rassure sur la permanence d'un monde toujours indemne. II projette une ligne d'audition contrôlable et reconnaissable à la manière d'un écran qui met fin à la turbulence et à la profondeur troublante du monde. Exercice de conjuration pour faire obstacle à la raréfaction du sens.

« II semble que le dernier reste de silence existant encore doive être refoulé, qu'ordre ait été donné d'arrêter le silence dans chaque homme et dans chaque maison, deletraiter en ennemi et de l'anéantir. Les avions battent le ciel à la recherche du silence qui campe derrière le nuage, les rafales des hélices sont comme des coups contre le silence », écrit M ax Picard. ${ }^{29}$

28. F. M urray-Schaffer nomme cette coupure une schizophonie ( voir F. M URRAY -SCHAFFER, Le paysage sonore, Paris, Lattès, 1979).

29. M ax PICARD, Le monde du silence, p. 295-296. 
Les zones desilencesont particulièrement vulnérables aux agressions sonores. Lemoindre bruit fait tache d'huile et pénètre delongues distances. Une scie électrique, une voiture ou une moto sur les routes de terre d'une forêt, un hors-bord sur une rivière ou un lac, brisent le charme des lieux en leur ajoutant un élément étranger que ces espaces ne peuvent intégrer. Ils lui portent atteinte car ils restreignent brutalement son usage en en faisant un simple résidu du bruit. Dans ces circonstances la contradiction est nette entre la nature et la technique. Là où le bruit est lié à la vitesse, à la puissance, à l'énergie, au pouvoir, le silence est à l'inverse une cristallisation de la durée, un temps arrêté ou infiniment lent, ouvert à la sensorialité du corps humain, battant au rythme tranquille de la marche del'homme. O n peut penser avec J acques Brosse qu' « il n'existe pas de bruit dans la nature, mais seulement des sons. $\mathrm{N}$ ullediscordance, nulle anarchie. M êmele grondement du tonnerre, mêmelefracas d'une avalanche ou la chuted'un arbre en forêt répondent à des lois acoustiques et ne les transgressent pas». ${ }^{30}$

Les entreprises de liquidation du silence foisonnent, elles ne sont pas délibérées, elles sont simplement le fait des conséquences de la technique. Elles ajoutent aux bruits de l'environnement urbain ou des innombrables instruments devenus indispensables à la vie sociale ou individuelle, investissent des lieux encore préservés, en friches, livrés à la pure gratuité du silence. La modernité traduit une tentative diffuse de saturation de l'espace et du temps par une émission sonore sans repos. Le silence étant une zone non défrichée, suspendue aux attentes, libre d'usage, il provoque une riposte de remplissage, d'animation, afin que soit enfin rompu ce défi de I'« inutile» qu'il recelait. Car pour une logique productive et marchande le silence ne sert à rien, il occupe un temps et un espace qui pourraient bénéficier d'une fin vouée à un meilleur rendement. Pour la modernité le silence est un résidu en attente d'un emploi plus fructueux, il est à l'image d'un terrain vague au coeur de la ville, une sorte de défi appelant à l'impératif de le rentabiliser, de lui faire rendre gorge d'une utilité quelconque puisqu'en attendant il n'est là qu'en pure perte. A nachronique, domaine où le bruit n'a pas encore pénétré, il est un archaïsme qui doit trouver son remède. II résonne comme une panne assourdissante du système. Le silence est un reste,

30. J. BR OSSE, Inventaire des sens, Paris, Grasset, 1965, p. 295-296. 
ce que le bruit n'a pas encore pénétré ni altéré, ce que les moyens ou les conséquences de la technique épargnent encore. ${ }^{31}$

\section{RÉSUMÉ}

Le silence essentiel à l'écoute nouvelle. $\mathrm{H}$ ors du bruit, du grondement d'une civilisation moderne, du tintamarrequotidien, il est un chemin vers l'écoute d'une humanité tapie au fond de l'être. Silence solitaire, silence partagé, silence lourd de sens, à contresens de ce qui voudrait nous éloigner de nousmêmes. Des mots à lire en silence pour se redonner des espaces où mieux se dire se conjugue avec mieux se taire.

\section{ABSTRACT}

Essential silence to a new listening. O utside the noise, the rumbling of a modern civilization, the daily uproar, there exists a path leading to the listening of humanity snuggled deep dow $n$ in every being. A solitary silence, a shared silence, a silence loaded with meaning which takes us in the opposite direction from that which pulls us apart. Words to be read in silence in order to restore spaces where a better communication goes hand in hand with being more silent.

31. Sur ce point et sur les aspects anthropologiques du silence nous renvoyons à D. LE BRETON, D u silence. 